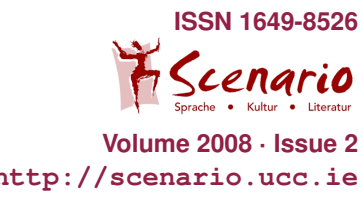

\title{
Romeo Juliet an der Realschule
}

eine Projektdokumentation

Jenny Passon

\begin{abstract}
Zusammenfassung
Shakespeare im Unterricht ist vor allem im Gymnasialbereich anzutreffen. Die Umsetzung von Shakespeares Stücken im Bereich der Sekundarstufe I wird trotz kreativer Ansätze weiterhin skeptisch betrachtet. Dieser Beitrag dokumentiert den Verlauf eines englischsprachigen Theaterprojekts, das an einer deutschen Realschule im Schuljahr 2007/2008 mit einer 10. Klasse durchgeführt wurde. Dabei werden zunächst die anvisierten Projektziele vorgestellt. Anschließend werden Kompetenzerwerb und Herausforderungen bezüglich der Sekundarstufe I diskutiert sowie Rahmenbedingungen und Interpretation der Shakespeare-Texte beschrieben. Der zweite Teil widmet sich der Projektdurchführung und beleuchtet exemplarisch ausgewählte Stunden. Der Beitrag wird mit einer Reflexion über Projektverlauf und Lernzuwachs abgerundet.
\end{abstract}

\section{Projektziele}

The theatre is so endlessly fascinating because it's so accidental. It's so much like life. (Arthur Miller)

Dieser Zusammenschluss - die Faszination Theater und das zufällige Leben - waren ausschlaggebende Antriebskraft für die Umsetzung des englischsprachigen Projekts ETC (English Theatre Class) an einer Realschule in Baden-Württemberg. ETC wurde während eines Schulhalbjahrs als eine wöchentlich zweistündige Arbeitsgemeinschaft realisiert. Es wurde offen und experimentell begonnen, da zunächst die Prozessorientierung des projektorientierten Unterrichts im Vordergrund stand (vgl. Apel/Knoll 2001: 54). Dieser experimentelle Einstieg ist auf die persönliche Laientätigkeit der Autorin im Bereich englischsprachigen Theaters sowie auf ihre positiven Erfahrungen mit dem bilingualem Regelunterricht zurückzuführen. Im Verlauf zeigte sich, dass die Schülerzentriertheit des Projekts in Bezug auf Kreativität, Unabhängigkeit, Motivation und Eigenverantwortung gewinnbringend für die Realschüler war. Nachträglich betrachtet liegt die besondere Chance derartiger Realschulprojekte m.E. aber vor allem auch im fremdsprachlichen Bereich. Der Rahmen von ETC bot 
sowohl leistungsstarken als auch leistungsschwachen Schülern einen Raum zum zwanglosen, mündlichen Gebrauch der Fremdsprache.

Allerdings wurden anvisierte, offene Projektziele formuliert. Zum einen sollte die Freude am gesprochenen Wort und an Formen des darstellenden Spiels vermittelt werden. Zum anderen sollten Gruppendynamik, Verantwortungsbewusstsein und Empathiefähigkeit gefördert werden. Gemäß des didaktischen und methodischen Prinzips des bilingualen Unterrichts wurde während der gesamten Probenzeit - beinahe nur „nebenbei“ - die so genannte „weitgehende Einsprachigkeit" (Ministerium Baden-Württemberg 2006: 9) angewandt. Im Laufe der Unterrichtsarbeit verlagerte sich der Schwerpunkt von der Prozesshin zu einer Produktorientierung. Zum Hauptziel der ETC wurde die zweifache Aufführung des Stücks in englischer Sprache sowie die Ermöglichung eines „englischen Erfolgserlebnisses“ für die Teilnehmer.

Die Fragestellung des Projekts lautete: Which steps must be taken to stage a play? Aufgrund dieser Frage entstanden reale Sprechanlässe, die hohe Eigeninitiative und die Fähigkeit zur Bewältigung echter Konflikte erforderte. Bezüglich des Einsatzes der Fremdsprache wurde während der gesamten Probearbeiten auf bewährte bilinguale Konzepte zurückgegriffen.

Die Funktion der Sprache im bilingualen Unterricht wird in der Handreichung Bilingualer Unterricht. Lernen für Europa folgendermaßen dargestellt:

Der Erwerb von Sprachkompetenz wird verstärkt als ganzheitliche Aufgabe gesehen. Bilingualer Unterricht bedeutet einerseits eine wesentliche Intensivierung des fremdsprachlichen Lernens und eine Ausweitung des fremdsprachlichen Handelns, bietet andererseits den Schülerinnen und Schülern die Chance, spezifische Sachgebiete mit Hilfe einer Zweitsprache zu erschließen und fachlich bedeutsame Inhalte und Problemstellungen unter neuem Blickwinkel zu erarbeiten und dadurch vielleicht sogar ihre Blickwinkel und ihre interkulturellen Handlungsmöglichkeiten zu erweitern. Darüber hinaus erleben die Schülerinnen und Schüler die fremde Sprache als wirkliches Kommunikationsmittel. (Ministerium Baden-Württemberg 2006: 6)

Natürlich spielte auch das fremdsprachliche literarische Lernen eine Rolle. Den Schülerinnen und Schülern wurde eine adaptierte Version von Shakespeares Romeo and Juliet zur Umsetzung und freien Interpretation überlassen. Voraussetzung dafür waren Textverständnis und die Erarbeitung von Figurenkonstellationen. Gemäß Burwitz-Melzer (2007: 138) sind hierzu die folgenden Fertigkeiten und Fähigkeiten als Teilkomponenten zur fremdsprachlichen Lesekompetenz relevant:

- motivationale Kompetenzen,

- kognitive und affektive Kompetenzen,

- interkulturelle Kompetenzen [...],

- Kompetenzen der Reflexion. 
Burwitz-Melzer (ibid.) räumt dabei ein, dass „diese Teilkomponenten als miteinander stark verwobene Fertigkeits- und Fähigkeitsbereiche zu sehen sind, die sich oft überschneiden." In der abschließenden Reflexion werden sie in Bezug auf ETC kommentiert.

Wie bereits erwähnt, erfüllte das Projekt trotz der anfangs untergeordneten Rolle dieses Aspektes, nachträglich betrachtet, einige wesentliche Aufgaben des Fremdsprachenunterrichts aus dem Bildungsplan 2004/Baden-Württemberg:

Zentrale Aufgabe des Englischunterrichts ist die Entwicklung kommunikativer Kompetenz, insbesondere die Förderung von Sprechkompetenz, Hör- und Leseverstehenskompetenz [...] Wichtiger als verfügbares Wissen ist die Befähigung der Schülerinnen und Schüler zu selbstständigem Handeln. (Ministerium Baden-Württemberg 2004: 72)

M.E. wäre es durchaus sinnvoll, in künftigen ETC-Projekten den fremdsprachlichen Aspekt noch weiter in den Vordergrund zu rücken. Dabei könnte die Theaterpädagogik konkret als Mittel genutzt werden, um die mündliche Ausdrucksfähigkeit der Schüler zu verbessern.

Ferner wurde Handlungsorientierung dadurch erreicht, dass die impulsgebende Lehrerin eine eher koordinierende Funktion übernahm. Dies gelang indem sie „zugunsten von zunehmend in selbstständiger Informationsbeschaffung und -verwertung eingeübten Lernern" (Petersohn/Volkmann 2006a: 9) in den Hintergrund trat. So konnten die Schüler ihre Kreativität durch schöpferische Aktivität, die Intellekt und Sinne weckt, erproben. Die von der Lehrkraft regelmäßig angeleitenden Warm-ups in Form von Pantomime, Standbildern, Positions- und Sprechübungen sollten den Schülern helfen, „kognitiv und emotional auf Texte reagieren zu können“ (ibid., Hervorhebung im Original). Dieser rezeptionsästhetische Ansatz bildet gemäß Petersohn/Volkmann einen Grundtenor fachdidaktischer und methodischer Überlegungen der letzten Jahre:

Der Unterricht geht dabei auf den Erfahrungshorizont der Schülerinnen und Schüler ein, wobei es zu einer der wichtigsten Aufgaben der Lehrkraft gehört, zwischen der Wertewelt des Textes und der der Lernenden gleichsam zu vermitteln. (ibid)

M.E. war das Verhältnis „Lehrer-Schüler“ insbesondere durch die Freude am Spiel geprägt. Die Schüler verstanden mich vorrangig als Expertin der Theaterpädagogik und nicht als Englischlehrerin. Obgleich sie gelegentlich bei Ausspracheschwierigkeiten an mich herantraten, nahmen sie mich nicht als notengebende Lehrperson wahr, sondern als Initiatorin der Arbeitsgemeinschaft ETC. Es war meine Absicht zu vermitteln, dass der Erfolg (bzw. Misserfolg) der Aufführung in den Händen der Schüler selbst lag. Aufgrund des zu Anfang vorgelegten engen Zeitplans - die Probearbeiten begannen Anfang September, die Premiere stand Ende November an - war die Mitarbeit aller Teilnehmer unabdingbar. Diese ihnen zugetragene Verantwortung erklärt möglicherweise die konzentrierte Arbeit während Scene Work und Performance Work sowie den Erfolg der Aufführungen. 
Burwitz-Melzer (2007: 138ff.) führt darüber hinaus an, dass der schulische Leseprozess dem privaten dahingehend möglichst angeglichen werden soll, „dass er verstärkt top-down-Leseverfahren einübt und in der Unterrichtsarbeit Platz lässt für spontane Reaktionen der Lernenden." Ferner bezeichnet Bredella (2002: 43) das top-down-Leseverfahren im Literaturunterricht als ein Lesen, das „von Erwartungen, dem Vorwissen und den Interessen des Lesers zu den einzelnen Gegebenheiten des Textes verläuft." Übertragen auf ETC bedeutete, dass die Schülerinnen und Schüler den Leseprozess von der Rollenfindung bis zur Präsentation des Stücks eigenständig gestalten sollten. Obgleich das Stück vorgegeben wurde, bot das Thema eine Vielzahl von Möglichkeiten für die Schüler, je nach Interesse und Einsatz, ihre personalen, sozialen, methodischen und fachlichen Kompetenzen zu vertiefen. Die fremdsprachlichen Sprechanlässe waren vielfältig und orientierten sich an den anvisierten Projektzielen. Angefangen mit der selbstständigen Rollenverteilung, über die Themenfindung der neuen Version, eigene Rollenerarbeitung, Titelsuche, Kostüm- und Requisitenwahl, Bühnenbildgestaltung, Werbung bis hin zur Organisation des Premierenabends konnten die Schüler ihren Neigungen entsprechend mitwirken. Andererseits war auch eine klare Strukturierung seitens der Lehrerin vonnöten, da es sich - wie Burwitz-Melzer treffend feststellt - bei Schülern der Sekundarstufe I um eine besonders schwierige Schulstufe handelt. Auch in der literaturdidaktischen Fachliteratur, die sich mit dem Fremdsprachenunterricht beschäftigt, würden „insbesondere Schüler der Haupt- und Realschulen, bisher stets vernachlässigt" (ibid. 2004: 213). Der unten dargestellte Verlauf des Realschulprojekts ist jedoch ein Beleg dafür, dass die englischsprachige Theaterarbeit mit gerade dieser Zielgruppe gewinnbringend und erfolgversprechend sein kann.

\section{Shakespeare in der Realschule}

\subsection{Kompetenzerwerb}

Folgende fachlichen Kompetenzen für Englisch (1. Fremdsprache = L2) wurden während des ETC-Projekts angebahnt, geübt, vertieft: Die Schülerinnen und Schüler können sowohl einfache als auch komplexe englische Äußerungen verstehen. Sie können Kontext, Gestik, Mimik, Stimmstärke und Intonation zum Sprechen und Verstehen nutzen. Sie können an Gesprächen teilnehmen und Meinungen äußern. Sie können einen auswendig gelernten Text mit rhetorischen Mitteln präsentieren und weitgehend die richtige Intonation anwenden. Die Schüler können eine Situation beobachten und kritisch-konstruktiv mit dem erlernten Fachvokabular Rückmeldung geben. Sie können einen Text von Shakespeare lesen und verstehen (vgl. Ministerium Baden-Württemberg 2004: 79ff.). 


\subsection{Herausforderung der Sekundarstufe I}

Shakespeare im Unterricht wird schon seit einigen Jahrzehnten in der literaturdidaktischen Fachliteratur diskutiert. Dabei bildete gemäß Petersohn/Volkmann das Didaktische Handbuch von Rüdiger Ahrens (1982) „lange Zeit für viele Lehrkräfte einen wichtigen Orientierungspunkt" (Petersohn/Volkmann 2006b: 7). Ahrens führt die Tradition der Shakespeare-Behandlung im Englischunterricht darauf zurück, dass seine Werke „in besonderer Weise mit bildungstheoretischen, politischen und literaturwissenschaftlichen Forderungen befrachtet worden" seien (Ahrens 1977: 13). Die Aufsätze von Ahrens (1977: 12ff.), Eisenmann (2007), Freifrau von Ledebur (1977: 63ff.), Hombitzer (1977: 77ff.) et al. thematisieren wiederum lediglich den Einsatz der Shakespeare-Themen im Bereich der Sekundarstufe II: „In den neusprachlichen Gymnasien der Bundesrepublik gehörte zumindest ein Shakespeare-Drama in der Originalsprache zum obligatorischen Lesestoff der Oberstufe" (vgl. Freifrau von Ledebur 1977: 63). 2006 konstatierte Ahrens jedoch einen Paradigmenwechsel:

Bei aller Disparatheit der [...] vorgetragenen Anregungen und wissenschaftlichen Erkenntnisse bleibt doch festzustellen, dass die Intensität der Behandlung von Shakespeares Dramen im Englischunterricht auf allen Schulstufen und in allen Schultypen eher zu- als abgenommen hat und dass man nach alternativen und kreativen Zugängen zu diesem Werk sucht, um den neuen pädagogischen und didaktischen Herausforderungen gerecht zu werden (Ahrens 2006: 107).

Ein Beispiel für einen solchen kreativen Zugang ist der Artikel von Lütge (2007), in dem keine expliziten Einschränkungen bezüglich der Alterstufe der Schüler gemacht werden. Nichtsdestotrotz erkennt Burwitz-Melzer eine Vernachlässigung der Haupt- und Realschulen in der literaturdidaktischen Fachliteratur, die sich mit dem Fremdsprachenunterricht beschäftigt. Sie führt den Verzicht auf die Auseinandersetzung mit der Sekundarstufe I auf das schwierige Übergangsstadium zurück, in dem sich die Schüler befinden:

Die seit Beginn der 80er Jahre erschienen Handreichungen und Materialien zum Einsatz von Literatur, die in der Regel große Praxisnähe und eine Fülle von kreativen Methoden in sich vereinen, weisen oft nur vereinzelte Vorschläge auf, um den Unterricht in der Sekundarstufe I abzudecken: Ihr Schwerpunkt liegt auf Unterrichtsvorschlägen für eine postpubertäre, leistungsstarke und lernwillige Klientel, die bereits über ausführliche Literatur- und Sprachkenntnisse verfügt (ibid. 2004: 214).

In diesem Sinne war das ETC-Projekt ein Versuch, auch Realschüler mit Shakespeare in Berührung zu bringen. Vorgabe für Realschulprojekte ist der Umgang mit Realsituationen. Sie sollen die Identifikation der Schüler mit ihrer Region stärken. ETC ermöglichte den Umgang mit der Realsituation, insofern als es die Interpretation einer neuen Version von Shakespeares Romeo and Juliet verlangte. Dazu war eine Auseinandersetzung mit der Region nötig. Entscheidend dafür war die Fragestellung: Which two fighting groups have always 
hated each other even though hardly anyone remembers why? Die Realsituation - Badener und Schwaben sind seit jeher „verfeindete“ Nachbarn - gab Anlass zur Diskussion - auf Englisch! Die aktuellen, gewaltbereiten Auseinandersetzungen zwischen den jugendlichen Fans der entsprechenden Fußballvereine - Karlsruher Sportclub (KSC) und VFB Stuttgart (VFB) - wurden thematisiert. Ferner wurden die Gründe für den anhaltenden Konkurrenzkampf von Badenern und Schwaben im historischen Kontext - erbitterte Kämpfe zwischen Befürwortern und Gegnern des geplanten „Südweststaats“ bereits im Jahre 1951 - gesucht. Ziel war es, zur Erweiterung des Erfahrungshorizontes der Schülerinnen und Schüler beizutragen. Die fremdsprachliche Literatur hat die Realschüler zum Nachdenken über ihre eigene Kultur und Identität angeregt und weitere fremdsprachliche Sprechanlässe geschaffen. Aufgrund dieser Vorgehensweise wurde auch ein wichtiger Bestandteil der zu entwickelnden interkulturellen Kommunikationskompetenz nach Byram - „Skills of interpretating and relating“ - (ibid. 1997: 52) angebahnt:

\begin{abstract}
Fertigkeit zu interpretieren und Texte in Beziehung zu setzen: Aufbauend auf dem Wissen über die eigene Kultur ist der intercultural speaker in der Lage, Texte oder Ereignisse aus der anderen Kultur zu erklären, zu interpretieren und zu ebensolchen Texten und Ereignissen in der eigenen Kultur in Beziehung zu setzen. Damit sind schriftliche, aber auch mündliche „Texte“ gemeint wie z.B. Gespräche oder Interviews (MüllerHartmann/Grau 2004: 2)
\end{abstract}

Entsprechend setzten die Realschüler den Shakespeare-Konflikt zwischen den Familien Montague und Capulet aus der anderen Kultur mit dem Konflikt zwischen Badenern und Schwaben in der eigenen Kultur in Beziehung. Denn zum Kennenlernen der eigenen und der fremden Kultur gehören gemäß Klippel (1991: 19) zweierlei Einsichten, „die, daß es im fremden Land anders zugeht als bei uns, und die, daß es gleiche Verhaltensformen, Werte und Einstellungen gibt." Als gleiche Verhaltensformen und Einstellungen sind die unerklärlichen Konflikte zweier Gruppierungen - Badener und Schwaben - zu begreifen, die aus der Geschichte entstanden sind und bis heute fortgeführt werden. Sicherlich kann diese Vorgehensweise aus theaterpädagogischer Sicht als ein gewöhnlicher Transfer begriffen werden. Die Gemeinsamkeiten bzw. Unterschiede zum Deutschen wurden nicht im historischen Kontext gesucht, was in diesem Rahmen auch wenig sinnvoll gewesen wäre. Hingegen regte Shakespeares englischsprachiger Plot die Schülerinnen und Schüler zum Nachdenken über die eigene Kultur und Identität an - m.E. ein wichtiger Schritt auf dem Weg zur interkulturellen Kompetenz. Der kritische Blick auf die eigene Kultur wurde dadurch geschärft, dass zumindest eine Bewusstmachung über den Hintergrund eines regionalen Konflikts entstand.

\title{
2.3 Rahmenbedingungen von ETC
}

ETC fand an der Alfred-Delp-Realschule (ADRS) in Ubstadt-Weiher statt; einer ländlichen Schule nahe Bruchsal in Baden-Württemberg. Das Projekt 
wurde im ersten Halbjahr des Schuljahres 2007/2008 wöchentlich 90 Minuten lang im Rahmen einer Arbeitsgemeinschaft durchgeführt. Teilnehmer waren 16-jährige Schülerinnen und Schüler der Klassen 10, die seit der 5. Klasse an der Realschule im Fach Englisch unterrichtet worden waren. Burwitz-Melzer hebt die allgemeinen Schwierigkeiten hervor, denen Literaturdidaktiker bei Schülern der Jahrgangsstufen 5 bis 10 begegnen:

- Die Lesekompetenz in der Mutter- oder Zweitsprache ist nicht sehr hoch und innerhalb der Jahrgänge sehr heterogen.

- Die Sprachkompetenz der Jugendlichen in der Zielsprache ist sehr heterogen.

- Die literarische Vorbildung der Jugendlichen ist heterogen, sie wird gerade in den Jahrgangsstufen 5 bis $10 \mathrm{im}$ Deutschunterricht erworben.

- Die Klassen 5 bis 10 umspannen mit Vorpubertät und Pubertät schwierige Phasen im Leben der Jugendlichen, die der Identitätsfindung dienen; ein Ausloten der heterogenen Interessenlagen der jugendlichen Mädchen und Jungen und eine motivierende Textauswahl wird besonders durch diese Tatsachen erschwert [...]. (ibid. 2004: 215)

Angesichts der genannten Schwierigkeiten bot der theaterpädagogische Ansatz von ETC im Umgang mit der fremdsprachlichen Literatur Möglichkeiten, differenziert vorzugehen. Da die Arbeitsgemeinschaft lediglich eine Lerngruppe von 15 Schülerinnen und Schülern umfasste, konnte beispielsweise während der Rollen- und Aufgabenverteilung die Heterogenität der Schüler bezüglich der Lesekompetenz, Sprachkompetenz, literarischen Vorbildung und zum Teil deren Entwicklungsstufe berücksichtigt werden.

Die Bedingungen für die Teilnahme am Projekt wurden den Schülern transparent gemacht. Der wichtigste Punkt war das Interesse und die Motivation, an einem englischsprachigen Theaterstück mitzuwirken. Nach dem ersten Treffen und der Einführung des Shakespeare-Plots entschieden sich 15 von 20 Schülern per Unterschrift zur Teilnahme. Dieser „Vertrag“ sollte eine Art Verbindlichkeit vermitteln und Übernahme von Verantwortung hervorrufen. Darüber hinaus wurde verdeutlicht, dass ETC eine Chance sei, Englischkenntnisse in einem notenfreien Raum zu vertiefen und sich somit auf die verpflichtende mündliche Abschlussprüfung vorzubereiten. ${ }^{1}$

Ferner wurde vorab betont, dass eine gute Englischnote keinesfalls Teilnahmebedingung sei. Der Fokus lag auf der Erarbeitung des Theaterstücks, während Englisch als Medium und somit als Arbeits- und Umgangssprache galt. Maßgebend war, wie im bilingualen Regelunterricht einen Spracherprobungsraum zu schaffen, in dem die Schülerinnen und Schüler angst- und sanktionsfrei die Fremdsprache anwenden und erproben:

\footnotetext{
${ }^{1}$ In der Realschule wird am Ende der Klasse 10 die Stufe B1 des Gemeinsamen Europäischen Referenzrahmens für Sprache: lernen, lehren, beurteilen erreicht (vgl. Ministerium BadenWürttemberg 2004: 72).
} 
Die Fehlerkorrektur der Lehrkraft orientiert sich an einem gentle feedback. Wenn eine Schülerin oder ein Schüler bei einem mündlichen Unterrichtsbeitrag ins Stocken gerät und die Lehrkraft schon ahnt, welches Wort fehlt, dann sollte sie spontan das Wort - in die Redepause der Schülerin/des Schülers hinein - zur Verfügung stellen (bridging/prompting). Wenn dies nicht geleistet werden kann, wird die Schülerin/der Schüler entweder das deutsche Wort im Beitrag benutzen oder die Lehrkraft um eine Übersetzung bitten (code-switching). (Ministerium Baden-Württemberg 2006: 14)

\subsection{Interpretation des Shakespeare-Stücks}

William Shakespeares Romeo and Juliet entstand gegen Ende des 16. Jahrhunderts und wurde erstmals 1623 in einer Gesamtausgabe publiziert. Es gibt seither viele Diskussionen und Produktionen, die das Stück auf die folgenden Aussagen reduzieren: „It is a 'play of young love' [...] 'the great typical love-tragedy of the world', and so on" (Taylor 1996: 7). Dennoch ist gemäß Taylor Romeo and Juliet mehr als eine altbekannte, dramatische Liebesgeschichte. Es ist ein Kunstwerk, das diverse Impressionen, Ideen, Vorstellungen und moralische Aspekte miteinander verknüpft. Vorzufinden sind unerwartet plötzliche Veränderungen wie die Umkehrung von Hass zur innigen Liebe. Über die Höhen der Liebe und die Tiefen der Trauer hinaus kommen Musik- und Tanzszenen vor sowie eine lebhafte Partyszene drinnen und eine romantische Mondszene draußen. Das Stück verkörpert Umstände und Situationen, die die Realschüler aus ihrer eigenen Lebens- und Erfahrungswelt kennen und nachvollziehen können:

Nothing in European drama had hitherto achieved the organization of so much human experience when Shakespeare, at about the age of thirty, undertook the story of Romeo and Juliet. (ibid. 1996: 7)

Diese Erfahrungen können auf den Alltag der Jugendlichen des 21. Jahrhunderts übertragen werden. Eine sprachlich simplifizierte Shakespeare-Adaption war Grundlage für die ETC-Version. Darüber hinaus wurden zur inhaltlichen Vereinfachung beispielsweise zwei Storytellers in die Handlung implementiert. Sie greifen kommentierend in das Geschehen ein und arrangieren diverse Zeitsprünge. Dennoch stimmen die Rahmenhandlung sowie die Figurenkonstellationen und -konflikte von William Shakespeares Original mit der Endfassung der ETC-Schüler überein.

Auch bei ETC treffen Romeo Montague und Juliet Capulet aufeinander. Deren Familien sind seit jeher miteinander verfeindet. Romeo, der eigentlich in Rosaline verliebt ist, wird von seinem Cousin und Freund Benvolio zu einem wagemutigen Besuch bei einer Capulet-Kostümparty überredet. Dort verliebt er sich Hals über Kopf in Juliet und vereinbart sogleich, mithilfe von Juliets Nurse, einen Hochzeitstermin bei Friar Lawrence. Unterdessen kommt es in der ETC-Version zu einem Straßenkampf zwischen den VFB-Capulets und den KSC-Montagues. Nach einem ausgiebigem Brainstorming und dem Wunsch das ShakespeareStück ins 21. Jahrhundert zu holen, einigten sich die Schüler aus aktuellem 
Anlass auf diese Fußball-Interpretation. Der VFB Stuttgart ist ein bekannter schwäbischer Fußballverein. Seine Fans sind, wie bereits oben angedeutet, aufgrund des andauernden schwäbisch-badischen Nachbarschaftskonflikts seit jeher mit denen des Karlsruher Sportclubs (KSC) verfeindet.

In der ETC-Inszenierung wurde dieser Streit der beiden Fußballfamilien schon mehrfach von Veronas - hier weiblichen - Herrschers Princess Escalus verurteilt. Dennoch wird Romeos Freund Mercutio bei einer gewalttätigen Auseinandersetzung von Juliets Cousin Tybalt umgebracht, woraufhin sich Romeo auf einen blutigen Kampf mit Tybalt einlässt. Die angestrebte dramaturgische Umsetzung des Kampfes soll an eine Schlägerei zwischen Hooligans erinnern. Als Juliet von Tybalts Tod und Romeos Verbannung nach Mantua hört, beschließt sie bei Friar Lawrence Rat zu holen. Nur er und die Nurse sind beiden Liebenden wohlgesonnen und hoffen, dass durch deren Vermählung der Streit der zänkischen Fußballclans beigelegt werden kann. Auch in der Interpretation der DELP-Schüler wird Romeo fälschlicher Weise von Abraham, einem KSCAnhänger, über Juliets Tod informiert. Das tragische Ende des Liebespaares ist allgemein bekannt. Doch die ETC-Mitglieder wollten dem Publikum gerade an dieser Stelle einen wichtigen Gedanken über den albernen Zwist zwischen KSC und VFB - und somit nicht zuletzt Badenern und Schwaben - auf den Weg mitgeben. Und so beenden sie das Stück mit dem chorisch gesprochenen Satz: Honestly, you don't even remember why!

\section{Projektdurchführung}

Abbildung 1 zeigt den Rehearsal Plan, der die jeweils aufgebrachte Zeit, Art des Warm-ups und Themenschwerpunkt enthält. Im Folgenden wird nun exemplarisch anhand von ausgewählten Probeterminen (in Abb. 1 fett gedruckt) die Umsetzung des Projekts skizziert.

Das erste Treffen galt als Einführung für die am Projekt interessierten Schülerinnen und Schüler. Dabei wurde ihnen die Struktur der Proben exemplarisch klar gemacht - Warm-up - Themenschwerpunkt - Cool-down. Letztlich sollten sie auch noch mit der Thematik bekannt gemacht werden und den ausgewählten Text kennen lernen, um anschließend eine Entscheidung über die Teilnahme am Projekt fällen zu können.

Für diese Phase war das wichtigste Ziel, die Thematik von William Shakespeares Stück Romeo and Juliet für die Schüler anschaulich und möglichst spannend zu gestalten. Bereits die Warm-ups motivierten die Schüler sehr. Nach einem persönlichen Kennenlern-Warm-up sollten sie nicht nur zum Sprechen, sondern auch in Bewegung gebracht werden. Dazu mussten die Schüler im Raum umherlaufen und auf ein Zeichen hin eine Person entsprechend der vorgegebenen Weise begrüßen: Walk around the room and say hi to someone $[\ldots]$ as a friend, [...] as someone you hate like an enemy, [...] as someone who is a member of your gang etc.

Die handlungsorientierte Texthinführung weckte ebenfalls ihr Interesse am Shakespeare-Plot und am Theaterspielen. Da das Stück bis zuletzt geheim blieb, 
Tabelle 1: Rehearsal Plan

\begin{tabular}{|c|c|}
\hline Week 1 (12.09.07) (90 min) & Introduction of ETC + Reading \\
\hline Week 2 (19.09.07) (90 min) & Warm-up: Voice $1^{\text {st }}$ Casting \\
\hline Week 3 (26.9.07) (90 min) & Warm-up: Body Language $2^{\text {nd }}$ Casting + Reading \\
\hline Week 4 (2.10.07) (90 min) & $\begin{array}{l}\text { Warm-up: Positions Scene Work + Scene } \\
\text { Performance }\end{array}$ \\
\hline Week 5 (10.10.07) (90 min) & $\begin{array}{l}\text { Warm-up: Facial Expressions Scene Work + } \\
\text { Scene Performance }\end{array}$ \\
\hline Week 6 (17.10.07) (90 min) & $\begin{array}{l}\text { Warm-up: Pantomiming Scene Work + Scene } \\
\text { Performance }\end{array}$ \\
\hline Week 7 (23.10.07) (90 min) & $\begin{array}{l}\text { Warm-up: Positions Scene Work + Scene } \\
\text { Performance }\end{array}$ \\
\hline \multicolumn{2}{|r|}{ FALL BREAK } \\
\hline Week 8 (6.11.07) (90 min) & $\begin{array}{l}\text { Warm-up: Character Building Scene Work + } \\
\text { Scene Performance }\end{array}$ \\
\hline $\begin{array}{l}\text { Week } 8 \text { (10.11.07) (4 x } 60 \text { min }) \\
\text { Rehearsal Saturday }\end{array}$ & Scene Work + Scene Performance \\
\hline Week 9 (15.11.07) (90 min) & Scene Work + Scene Performance \\
\hline Week 9 (16.11.07) (4 x 60 min) & $\begin{array}{l}\text { Warm-up: Voice + Body Language Scene Work + } \\
\text { Scene Performance }\end{array}$ \\
\hline Week 9 (17.11.07) (4 x $60 \mathrm{~min})$ & $\begin{array}{l}\text { Warm-up: Pantomiming Run-through + Curtain } \\
\text { Call }\end{array}$ \\
\hline Week $10(22.11 .07)(2 \times 60 \mathrm{~min})$ & Dress Rehearsal \\
\hline $\begin{array}{l}\text { Week } 10(23.11 .07+24.11 .07) \\
(2 \times 90 \mathrm{~min})\end{array}$ & Opening Night + Performance Night \\
\hline Week 11 (5.12.07) (90 min) & Project Evaluation \\
\hline
\end{tabular}

trafen alle Schüler in dieser Sitzung zum ersten Mal auf die ETC-Adaption von Romeo and Juliet. Per Zufallsprinzip wurden während der Text-Hinführung blaue Kärtchen (für Montague-Rollen) und rote (für Capulet-Rollen) sowie grüne für neutrale Rollen (z.B. Storyteller) mit jeweils stichwortartiger Rollenbeschreibung verteilt. Anschließend sollten sich die Schüler mit ihren Clanmitgliedern (rot, blau oder grün) auf einer Seite positionieren. Jeder Schüler stellte daraufhin seine Rolle vor und hatte somit seinen ersten kleinen Auftritt.

Für diese Phase war das wichtigste Ziel, die Projektidee für die Schüler anschaulich und möglichst spannend zu gestalten. Anschließend wurden die ersten Szenen in den zuvor verteilten Rollen gelesen. Bei Unterzeichnung des ETC-Vertrags erhielten sie jeweils ein Book bzw. Skript, um sich bis zum nächsten Treffen mit dem Gesamttext vertraut zu machen.

Schwierigkeiten beim Textverständnis der adaptierten Version wurden gelöst, indem nachträglich weitere Kürzungen vorgenommen und mehr Wortschatzangaben gemacht wurden. 15 Schüler (davon drei Jungen) meldeten sich verbindlich für das ETC-Projekt an. 


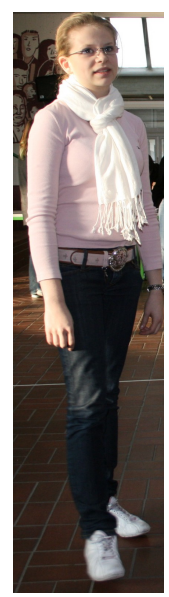

Hi! My name is Juliet and I am from the House of Capulet. In the play I don't want to get married to dad's friend Paris. When I meet Romeo at a Costume party, we fall in love. The problem is: he's the son of my enemy! You are Juliet (House of Capulet)! - don't want to get married to dad's friend Paris -meet Romeo at a Costume party: LOVE -problem: Romeo = son of enemy

Abbildung 1: Rollenkärtchen und improvisierter Auftritt von Juliet (später Storyteller)

In der zweiten Woche fand eine abgewandelte Form eines Castings statt. Zur Auflockerung wurde dieses Treffen mit Warm-ups zum Thema Voice begonnen. Es wurden eine Reihe Zungenbrecher auf den Tageslichtprojektor gelegt und die Schüler sprachen sie chorisch mit zunehmender Geschwindigkeit nach. Dabei stand vor allem der Faktor Spaß im Vordergrund. Ferner feilten sie dabei an ihrer Aussprache und konnten sich allmählich vom Schulalltag trennen und auf das Theaterspielen einlassen. Ein weiterer Schwerpunkt war die Bewusstmachung der Tonlage - Pitch. Für diese Übung stellten sich die Schülerinnen und Schüler im Kreis auf. Die Aufgabenstellung lautete: Point at the person at your right and say "you" as if you were [...] very angry at him/her, [...] disappointed with him/her, [...] in love with him/her etc. Anfangs zum Teil gehemmt - da nicht alle Schüler Erfahrungen im Bereich Theater hatten - ließen sich die Schüler zunehmend auf dieses Sprachspiel ein und gewannen große Freude daran. Dieses Warm-up lässt sich beliebig mit Sätzen aus dem Stück erweitern und wurde auch an anderen Stellen zur Bewusstmachung der Stimme wiederholt eingesetzt.

Zwar gab die Lehrerin die Vorgehensweise der Rollenverteilung vor, doch wurde sie weitestgehend selbstständig von den Schülern durchgeführt. Dafür schrieben sie zunächst zwei für sie denkbare Rollen mit Begründung auf ein leeres Blatt. Danach legten sie die Blätter offen auf den Boden und bekamen Zeit, die Wünsche der anderen Gruppenmitglieder in Stille zu lesen. Die Entscheidungen wurden im Unterrichtsgespräch - stets auf Englisch - getroffen. Dabei wurden die Rollen nacheinander vergeben und jeweils durch demokratische Abstimmungen bestätigt. Bei Doppelwünschen wurden die jeweiligen Teilnehmer angehört und durch Gruppenabstimmung eine vorläufige Entscheidung gefällt. Die Lehrerin nahm dabei lediglich die Rolle einer Gesprächsleiterin ein.

Die großen Rollen des Romeos und der Juliet wurden schnell und konfliktlos verteilt. Für die Rolle der Storyteller gab es mehrere Bewerber, die nach etwas län- 
geren Argumentationsphasen und Gruppenabstimmungen festgelegt wurden. Größere Schwierigkeiten gab es bei der Besetzung der Lady Capulet, da sich drei Schülerinnen dafür bewarben. Ein unbetroffener Schüler - nicht die Lehrkraft schlug vor, die drei Bewerberinnen selbst entscheiden zu lassen und der Gruppe ihren Entschluss beim nächsten Treffen mitzuteilen. Die Gruppenmitglieder waren damit einverstanden. In der nächsten Probe teilten jene Schülerinnen der Gruppe ihre Entscheidung mit. Obwohl erkennbar war, dass zumindest nicht alle Betroffenen zu 100\% mit der Entscheidung einverstanden waren, gab es dennoch - zumindest vordergründig - diesbezüglich keine offenen Konflikte. Den beiden übrigen Lady Capulet -Anwärterinnen wurden Doppelrollen nahe gelegt: Friar Lawrence/Princess Esculus und Mr Capulet/Mercutio.

Für die Intensivphase der Projektdurchführung waren fünf 90-minütige Proben und ein Rehearsal Weekend ( 8 x 60 Minuten) vorgesehen. In dieser Zeit sollten die Schüler die übrigen Szenen in Gruppenarbeit anlegen und gestalten. Zur Erarbeitung der Szenen wurden die Schüler in zwei Gruppen eingeteilt. Die Scene Works dauerten jeweils zwischen 15 und 30 Minuten. Bei Fehlen von Schülern mussten Rollen ersetzt werden. Somit war die Vorabplanung der zu bearbeitenden Szenen nur bedingt möglich. Nach jeder Phase der Scene Work stand entsprechend eine Scene Performance an. Die Gruppen kamen zusammen, um sich gegenseitig Feedback zu geben und Verbesserungsvorschläge zu machen - auf Englisch! Wichtig war in dieser Phase, dass die Schüler lernten, die in den Warm-ups erarbeitenden Theatertechniken in ihre Arbeit einfließen zu lassen und ein Gruppengefühl zu entwickeln. Außerdem sollten sie lernen, konstruktiv Rückmeldung zu geben und Figurenauslegungen von anderen zu akzeptieren. Exemplarisch für alle ETC-Proben während der Durchführungsphase steht die WEEK 7.

Thema des Warm-ups war Positions. Hierbei wurden die Schüler durch die Lehrkraft mit den Positionen full back, three quarters left/right, one quarter left/right, full front sowie shared positions vertraut gemacht. Anschließend sollten die Schüler in Partnerarbeit jeweils zwei zentrale Sätze der Rollen herausgreifen und zwei der zuvor eingeführten, zu den Sätzen passenden, Positionen ausprobieren (Abb. 3 und Abb. 4).

Später sollten sie diese der Gruppe vorführen, um zu prüfen, ob die Position gut gespielt und sinnvoll gewählt wurde. Ziel war, das Character Building durch bewusste Einfühlung in die Haltung der Rolle zu erfahren, wobei die Körpersprache im Zentrum stand.

Nach der Szenenerarbeitung stand die Titel- und Themenspezifizierung des Stücks an. Eine Schülerin stellte die gesammelten Titel für das Stück vor und leitete eine kurze Diskussionsphase ein. Letztlich wurden drei Titel zur Wahl gestellt:

1. Romeo \& Juliet - cool version -

2. Romeo \& Juliet - new version -

3. Romeo \& Juliet. Old (love) story - New version. 


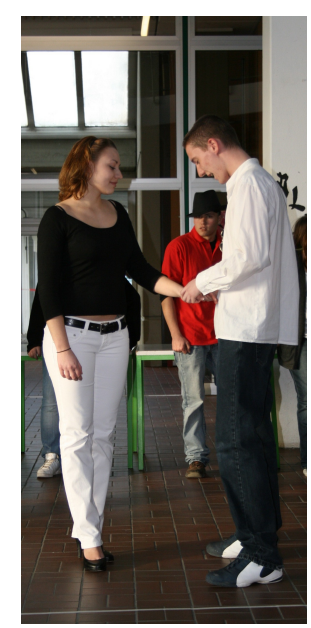

Abbildung 2: Shared position. Zentraler Satz von Romeo: Your hand is so soft, your lips red as roses.

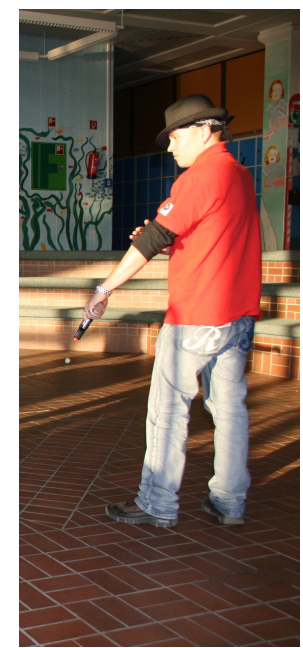

Abbildung 3: Three quarters right. Zentraler Satz von Tybalt: So, draw your sword and fight!

Die Gruppe kürte den dritten Vorschlag zum Titel. Anschließend kam es zur Diskussion über die Themenspezifizierung. Eine weitere Schülerin erläuterte die unterschiedlichen Themenvorschläge. Letztlich wurde der endgültige Entschluss getroffen, dass das Thema der neuen Version von Romeo and Juliet den Konflikt zwischen Badenern und Schwaben zeigen sollte. Zur Kennzeichnung der verfeindeten Familien bzw. Fußball-Fans diente die Farbe blau für die Montagues und die Farbe rot für die Capulets.

An dieser Stelle ist anzumerken, dass die Schüler während der Scene Works, die ohne ständige Anwesenheit der Lehrerin stattfand, gelegentlich ins Deutsche als Arbeitssprache wechselten. Während der gemeinsamen Scene Performances jedoch hielten sich die Schüler größtenteils an die Arbeitssprache Englisch. Möglicherweise lag das daran, dass die Schüler das englische Fachvokabular aus 
den Warm-ups kannten. Sie setzten deswegen eher Englisch bei Rückmeldungen an die Gruppenmitglieder ein als nach deutschen Fachtermini zu suchen. Schließlich war auch der Text auf Englisch. Nur wenn die Schüler vor Ideen übersprudelten, fielen sie ins Deutsche zurück und nutzen die Möglichkeit des code-switching. So sagte z.B. eine Schülerin, die sich mit der Rollenerarbeitung der Nurse beschäftigte: I don't know how to say this in English, but I could come onstage with a "Staubwedel“ and clean the audience. Die Lehrerin zog sich während der Intensivprobenphase (ab WEEK 8) als Director zunehmend zurück und machte allenfalls auf gravierende Aussprachefehler aufmerksam.

Ferner zeigten sich die Schülerinnen und Schüler in dieser Phase sehr kooperativ und flexibel, indem sie verantwortungsbewusst Rollen von fehlenden Schülernübernahmen. Es gelang den Schülern zunehmend besser, Rückmeldung auf eine Scene Performance zu geben, z.B.: We can only see your back. - Find another position! [...] Speak up. We can't understand what you are saying [...].

Die Projektdurchführung ist insgesamt zufrieden stellend abgelaufen - die Schülerinnen und Schüler arbeiteten sehr engagiert, motiviert und eigenständig. Eine große Schwierigkeit war jedoch, dass trotz der Anwesenheitspflicht die Gruppe selten ganz vollzählig war. In mehreren Metainteraktionen wurde das Problem thematisiert und nach Lösungsmöglichkeiten gesucht.

Es war zu beobachten, dass die Schülerinnen und Schüler sich gegenseitig bei der Rolleninterpretation und Szenenentwicklung halfen. Sie organisierten sich so, dass sie gegen Ende der Probenzeit weitestgehend eigenständig Feedback geben konnten. Darüber hinaus regelten die Gruppenmitglieder intern die Organisation der Requisiten auf vorbildliche Weise. Ihr Verantwortungsbewusstsein für die Gegenstände zeigte sich auch darin, dass vor den Präsentationen Kostüme und Requisiten stets bereit lagen. Es ist diesbezüglich anzumerken, dass Kostüme und Requisiten während der Erarbeitung eine untergeordnete Rolle spielten. Der theaterpädagogische Fokus lag eindeutig auf der Rollenerarbeitung und (Körper-) Sprache.

Weiter war zu beobachten, wie jeder einzelne Schüler entsprechend seiner Fähigkeiten tatsächlich Herz, Kopf und Hand anlegte, um das Projekt zum Erfolg zu führen. Herz, weil die Schüler Empathie bei der Rollenfindung und völlige Hingabe zum Theaterspiel zeigten sowie Verantwortungsbewusstsein im Umgang miteinander. Kopf, weil sie versuchten, beim Anlegen der Szenen die erlernten Theatertechniken zu integrieren und dabei bemüht waren, die englische Sprache möglichst perfekt einzusetzen. Und letztlich Hand, weil sie Kostüm- und Requisitenwahl, Werbung und Organisation des Premiereabends in Eigenregie gestalteten.

Die Opening Night und auch die zweite Performance Night wurden ein großer Erfolg. Es kamen an beiden Abenden etwa 100 Zuschauer, die die Arbeit der ETC-Truppe mit viel Applaus und Lob würdigten. Im Publikum vertreten waren sowohl Eltern, Schüler, Lehrer als auch auswärtige Interessierte, die durch die Bekanntmachung der Bruchsaler Neuesten Nachrichten (BNN) auf die Inszenierung aufmerksam gemacht worden waren. Einige Schüler der 8. Klassen hatten sich zuvor im Regelunterricht mit dem Plot vertraut gemacht 
und schrieben nach der Aufführung begeisterte Reports über die Inszenierung. Besonders die mündlichen Sprachfähigkeiten und die Interpretation (VFB-KSC) wurden dabei gelobt. Die schauspielerischen Fähigkeiten wurden differenziert und kritisch bewertet. Während die Storytellers viel Lob und Anerkennung für ihre beeindruckende schauspielerische Leistung sowie für den Aufwand beim langen, englischsprachigen Textlernen ernteten, kritisierten die Schülerreporter Lady Capulet und Mercutio als nicht überzeugende Darsteller. Auch Lehrerkollegen, die die Proben von ETC aus den von Burwitz-Melzer genannten Gründen (vgl. 2.3) zuvor sehr kritisch beäugt hatten, waren überrascht und begeistert vom Talent und vom Engagement ihrer Schüler. Besonders beeindruckt zeigten sich einige über die Entwicklung der als leistungsschwach und introvertiert bekannten Schüler, so z.B. den Darstellern des Romeo und des Mercutio sowie der Lady Capulet und der Nurse. Letztlich zog ebenfalls die Presse eine durchweg positive Bilanz über das Ergebnis des Realschulprojekts:

Mit viel Einfühlungsvermögen, großartigen schauspielerischen Fähigkeiten und vor allem einer bravourösen sprachlichen Leistung hatten die 15 Zehntklässler der ETC die tragische Liebesgeschichte von Romeo und Julia erarbeitet und umgesetzt. (BNN/psp 2007: 18)

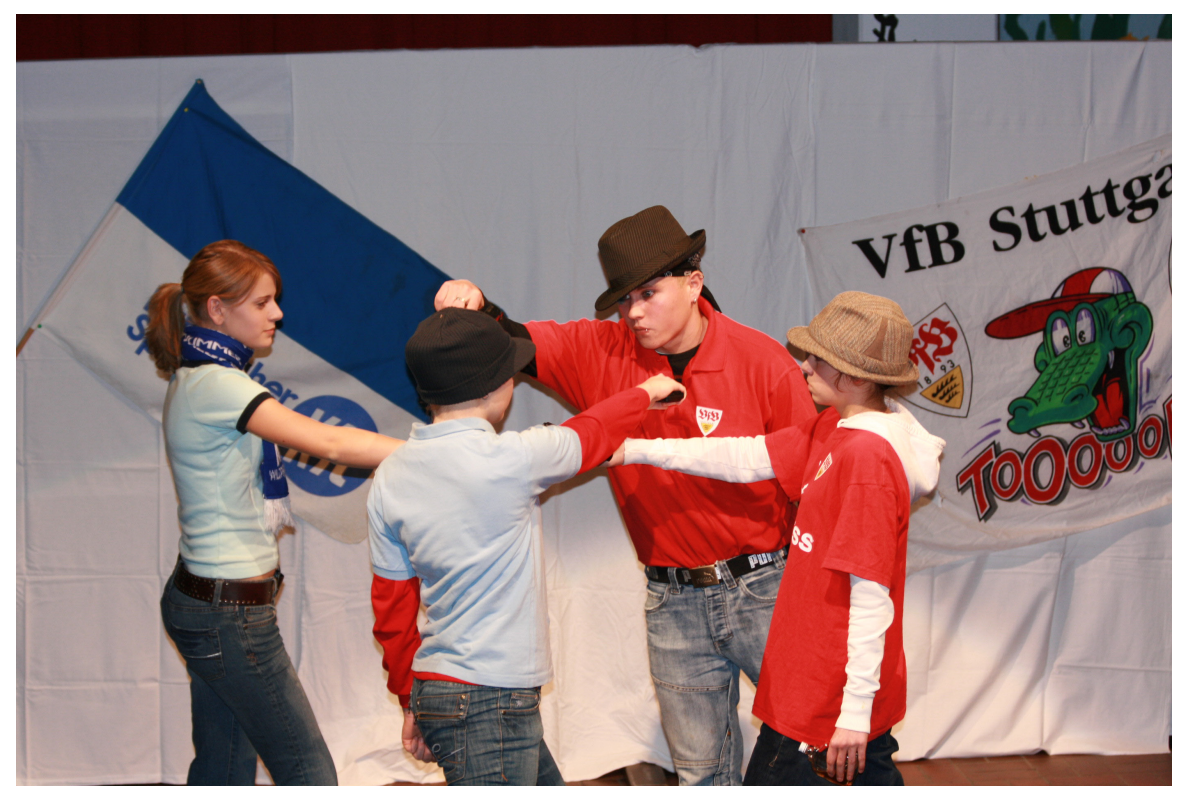

Abbildung 4: Kampfszene zwischen Montagues und Capulets

Während der Projektevaluation rekapitulierten die Schüler zunächst im offenen Gespräch den Verlauf des ETC-Projekts. Sie stellten fest, dass die Kooperation in einer neu zusammen gestellten Gruppe gut geklappt hatte und reflektierten, dass zukünftig eine anfänglich genauere Planung und generell mehr Probenzeit Fehler und Zeitdruck vermeiden würden. Insgesamt zeigten sich die Schülerinnen und Schüler sehr zufrieden und stolz über das Geleistete. Auf einem vorgefertigten Evaluationsbogen machten sie anonym 


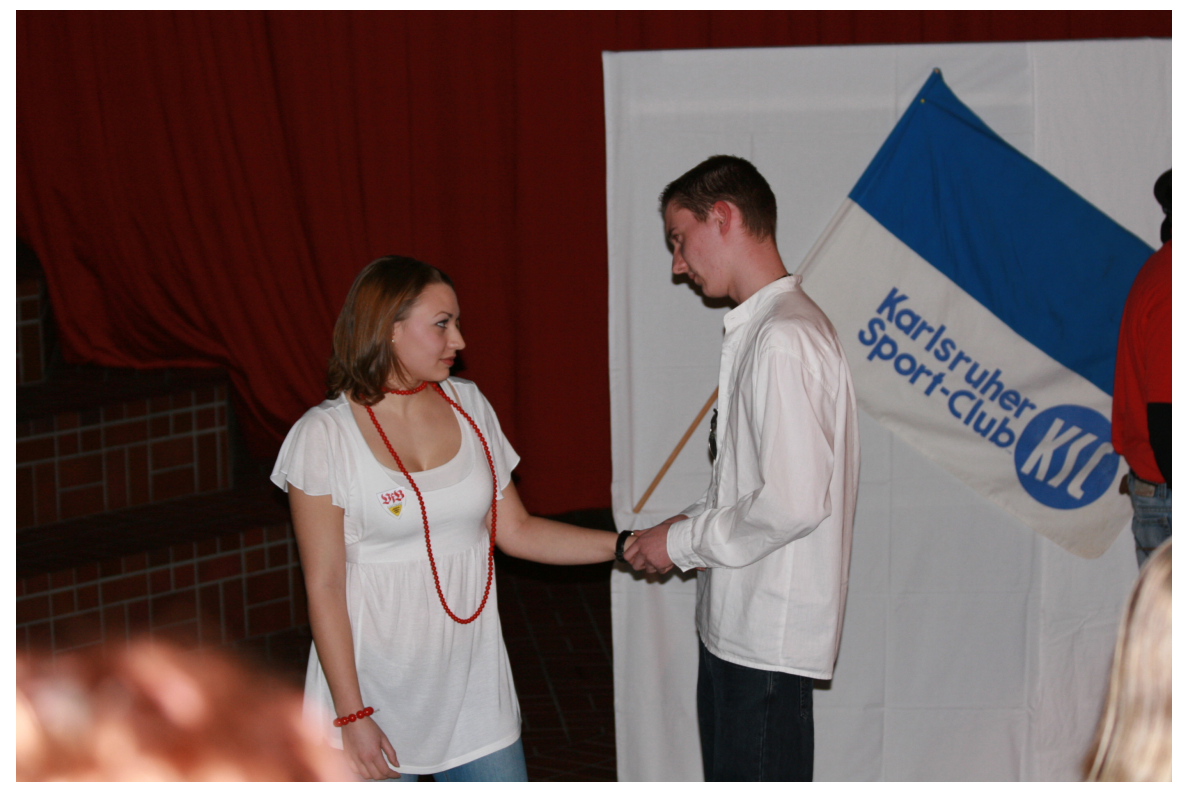

Abbildung 5: Liebeserklärung - Romeo und Juliet

schriftliche Angaben zum Lernzuwachs in den unterschiedlichen Bereichen. Die Auswertung ergab insgesamt eine sehr positive Bilanz des Projekts. In Bezug auf den Lernzuwachs bei ETC kreuzten elf von 15 Schüler den Bereich Englisch, acht den Bereich Theater und bemerkenswerterweise auch sieben Schüler den Bereich Selbstbewusstsein an. Bezüglich des literarischen Lernens gaben 14 von 15 Schülern an, den Text und Figurenkonstellationen komplett verstanden zu haben. Nur ein Schüler kreuzte zum Teil an.

Aus Sicht der Lehrkraft ist vor allem hervorzuheben, dass ETC ein differenziertes Lernen ermöglichte. Das zeigt zum einen die Tatsache, dass aus einer heterogenen, neuen Schülergruppe am Ende eine ETC-Gemeinschaft wurde. Zum anderen war während des Prozesses zu beobachten, dass sowohl leistungsstarke und extrovertierte Schüler (z.B. Darsteller der Storytellers und Juliet) als auch mäßig leistungsstarke und introvertierte Schüler (z.B. Lady Capulet) individuelle Fortschritte in den unterschiedlichen Bereichen - Theater - Fremdsprache - Selbstbewusstsein vorwiesen. Ganz erstaunliche Entwicklungen machten aber auch eher leistungsschwache und als problematisch geltende Schüler. Der Darsteller des Romeo, der als eher unzuverlässig und instabil galt, machte bereits während der zweiten Sitzung deutlich, dass er in der Romeo-Rolle eine Chance für sich sah. Während der Probenarbeiten steigerte er sich kontinuierlich im Bereich Fremdsprache und Theater. Als der Aufführungstermin näher rückte, machte ihm jedoch das Lampenfieber zu schaffen. Es gelang ihm dennoch - m.E. maßgeblich aufgrund der moralische Unterstützung und durch gutes Zureden der Gruppe - in beiden Aufführungen $\mathrm{zu}$ brillieren. Es kann also in diesem Fall geschlussfolgert werden, dass sein Selbstbewusstsein durch die Arbeit bei ETC gestärkt wurde. Ferner konnte er in der oben genannten mündlichen Englischprüfung eine deutlichen Leistungsan- 
stieg vorweisen. In einem Gespräch machte er der Lehrkraft deutlich, dass er die mündliche Leistungssteigerung auf den Lernzuwachs durch ETC zurückführte.

\section{Reflexion}

Zusammenfassend ist festzustellen, dass auch in der Sekundarstufe I der Umgang mit fremdsprachlicher Literatur durch einen theaterpädagogischen Ansatz möglich und gewinnbringend ist - trotz Heterogenität der Lerngruppe und trotz des schwierigen Übergangsstadiums der pubertierenden Jugendlichen. Um dies zu belegen, sollen an dieser Stelle noch einmal die von Burwitz-Melzer (2007: 138) genannten, relevanten Teilkomponenten zur fremdsprachlichen Lesekompetenz herangezogen werden. Folgende Kompetenzen wurden m.E. angebahnt, geübt bzw. vertieft:

- motivationale Kompetenzen, da die Schüler auf freiwilliger Basis der Arbeitsgemeinschaft beitraten und bereit waren selbstständig auch in ihrer Freizeit zu arbeiten;

- kognitive und affektive Kompetenzen, da die Schüler fremdsprachliches Textverständnis bezüglich der Figurenkonstellationen erwarben, um mit Hilfe von Empathie und Perspektivenwechsel ihre Rolle zu erarbeiten;

- interkulturelle Kompetenzen, da sie Probleme aus der anderen Kultur mit denen der eigenen in Beziehung setzten und somit ihr Bewusstsein für einen regionalen Konflikt geschärft wurde;

- Kompetenzen der Reflexion, da sie regelmäßig Feedback nach Scene Performances gaben und sich nach den Aufführungen kritisch zum Ablauf äußerten sowie ihren Lernerfolg schriftlich in einem Evaluationsbogen dokumentierten.

Ferner sei noch einmal hervorgehoben, dass der projektorientierte Unterricht in einem notenfreien Rahmen stattfand. Folglich konnten die Teilnehmer negative Auswirkungen auf Noten von Vornherein ausschließen und waren dadurch weniger gehemmt die englische Sprache mündlich einzusetzen. Zudem erhielten sie am Schuljahresende ein personalisiertes Testat für ihre Bewerbungsmappe. Diese Fakten trugen m.E. zum Erfolg des Realschulprojekts bei. Darüber hinaus gab es wesentliche Besonderheiten von ETC, die den Unterschied zu ähnlichen Projekten in der Sek. II verdeutlichen. Beispielsweise hat meiner Wahrnehmung zu Folge trotz allgemeiner Schwierigkeiten, denen Literaturdidaktiker bei diesem Schülerklientel begegnen, ein Lernzuwachs in unterschiedlichen Bereichen stattgefunden (vgl. ibid. 2004: 215):

- Trotz heterogener Lesekompetenz in der Mutter- oder Zweitsprache sowie heterogener literarischer Vorbildung der Jugendlichen, wurde der Inhalt aller Schüler erfasst. (vgl. Anhang, Schaubild 7) 
- Trotz heterogener Sprachkompetenz der Jugendlichen in der Zielsprache, gaben alle Schüler an „viel“ oder „sehr viel“ Englisch gehört und gesprochen zu haben. (vgl. Anhang, Schaubild 6)

- Trotz Pubertät als schwierige Phase im Leben der Jugendlichen, die der Identitätsfindung dient, bot ETC den Schülern Möglichkeiten gemäß ihrer heterogenen Interessenlagen eine individuelle Nische zu finden, um sich im Projekt einzubringen. (Burwitz-Melzer 2004: 215)

Die in der Fachliteratur häufig geäußerte Sorge, die Zielgruppe der Sekundarstufe I sei noch nicht in der Lage selbstständig zu agieren, konnte somit am Beispiel von ETC widerlegt werden. Nachahmern ist zu empfehlen, etwaige Irritationen wie das Thema, Verbindlichkeit' mit den Schülern vorab zu klären. Es ist beispielsweise möglich, durch einen Vertrag die Unzuverlässigkeit in einem gewissen Maße einzugrenzen. Durch Abgabe von Verantwortung und Vertrauen an die Schüler waren die Teilnehmer von ETC zu ganz Außergewöhnlichem fähig. Das Vertrauen in die Schüler, in ihre Fähigkeiten und in ihren Ideenreichtum ist nötig, um ein Erfolg mit ETC zu erzielen. Bezüglich der Fremdsprache ist außerdem eine konsequente Einsprachigkeit seitens der Lehrkraft erforderlich. Ferner war zu beobachten, dass die persönliche Theaterfaszination - wenn authentisch - auf die Schüler übertragbar ist. Abschließend ist zu sagen, dass in ähnlichen Realschulprojekten verschiedene Schwerpunkte gesetzt werden können. Es könnte beispielsweise durchaus sinnvoll sein, die Klassenstufen 8-9 einzubeziehen. Ferner ist denkbar, dem mündlichen Gebrauch der Fremdsprache während des Projektprozesses mehr Gewicht zu geben.

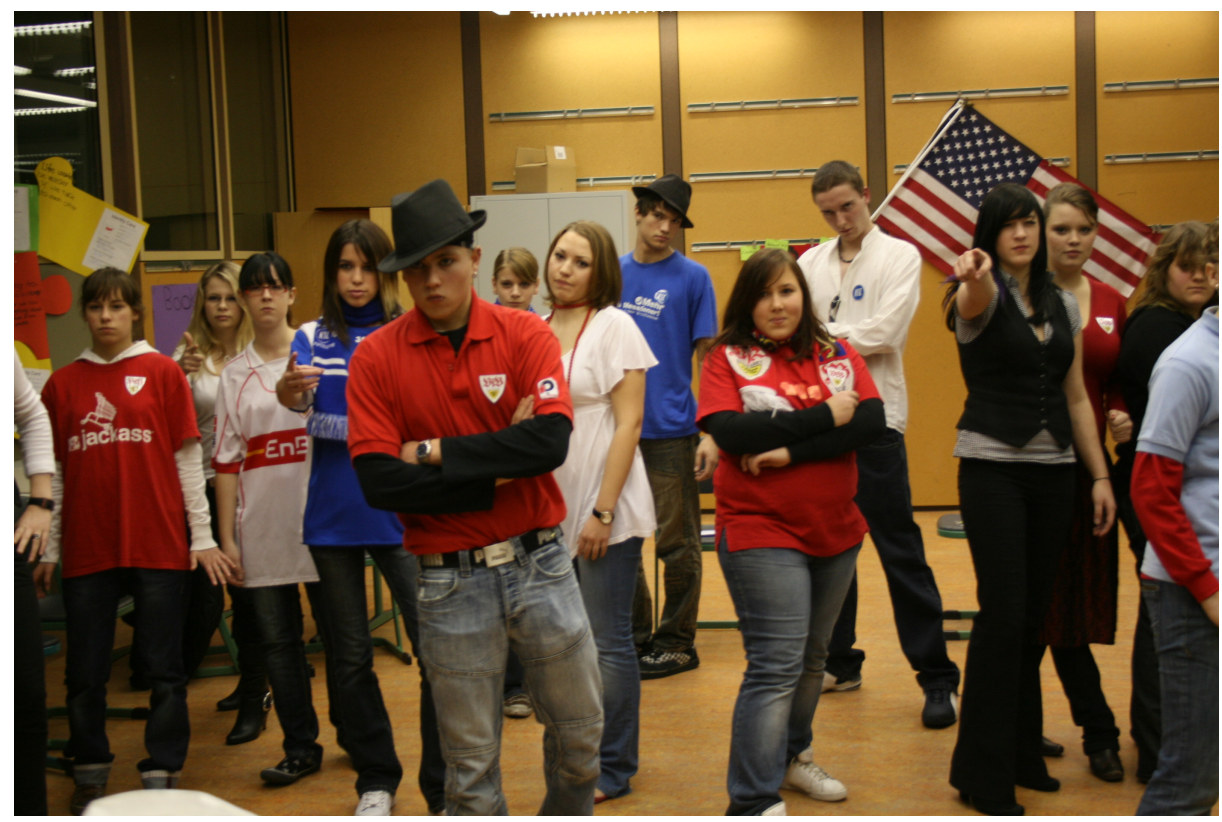

Abbildung 6: Warm-up vor der Premiere 


\section{Bibliographie}

Ahrens, Rüdiger (1977): Anglistik \& Englischunterricht. Shakespeare im Unterricht. Trier: Volksfreund-Druckerei

Ahrens, Rüdiger (1982): Shakespeare. Didaktisches Handbuch. Bd. 1-3. München: Flink-Verlag

Ahrens, Rüdiger (2006): Shakespeare adaptiert. In: Petersohn, Roland/Volkmann, Laurenz: Shakespeare didaktisch I. Neue Perspektiven für den Unterricht. Tübingen, Stauffenburg Verlag, 105-118

Apel, Hans Jürgen/Knoll, Michael (2001): Aus Projekten lernen. München: Oldenbourg-Schulbuchverlag

BNN/psp (2007): Fußball und Shakespeare? In: Bruchsaler Neueste Nachrichten (BNN) Ausgabe Nr. 275, 18

Bredella, Lothar (2002): Wie sollen wir literarische Texte lesen? Überlegungen zum „guten Leser“. In ders.: Literarisches und interkulturelles Verstehen. Tübingen: Gunter Narr Verlag, 34-79

Bredella, Lothar (2002): Literarisches und interkulturelles Verstehen. Tübingen: Gunter Narr Verlag

Bredella, Lothar/Burwitz-Melzer, Eva (2004): Rezeptionsästhetische Literaturdidaktik. Mit Beispielen aus dem Fremdsprachunterricht Englisch. Tübingen: Gunter Narr Verlag

Bredella, Lothar/Hallet, Wolfgang (Hrsg.) (2007): Literaturunterricht, Kompetenzen und Bildung. Trier: WVT

Burwitz-Melzer, Eva (2004): Lehrende und Lernende im fremdsprachlichen Literaturunterricht: In dies.: Rezeptionsästhetische Literaturdidaktik. Mit Beispielen aus dem Fremdsprachunterricht Englisch. Tübingen: Gunter Narr Verlag, 201-233

Burwitz-Melzer, Eva (2007): Ein Lesekompetenzmodell für den fremdsprachlichen Literaturunterricht. In: Bredella, Lothar/Hallet, Wolfgang (Hrsg): Literaturunterricht, Kompetenzen und Bildung. Trier: WVT, 127-158

Byram, Michael (1997): Teaching and Assessing Intercultural Communicative Competence. Clevedon et at.: Multilingual Matters

Eisenmann, Maria (2007): Shakespeares Hamlet im Englischunterricht der gymnasialen Oberstufe. In: Schewe Manfred/Even Susanne: Scenario Jahrgang 2007. Ausgabe 01. ISSN 1649-8526

Freifrau von Ledebur, Ruth (1977): Die Shakespeare-Lektüre in den Curricula der reformierten Oberstufe. In: Ahrens, Rüdiger: Anglistik \& Englischunterricht. Shakespeare im Unterricht. Trier: Volksfreund-Druckerei Nik. Koch, 63-76

Hombitzer, Eleonore (1977): Shakespeare-Lektüre in der reformierten gymnasialen Oberstufe - unter besonderer Berücksichtigung von 
Grundkursen. In: Ahrens, Rüdiger: Anglistik \& Englischunterricht.

Shakespeare im Unterricht. Trier: Volksfreund-Druckerei Nik. Koch, 77-92

Klippel, Friederike (1991): Zielbereiche und Verwirklichung interkulturellen Lernens im Englischunterricht. In: Der fremdsprachliche Unterricht 1, 15-21

Lütge, Christiane (2007): 'And lose the name of action'? Überlegungen zur Schüleraktivierung mit Drama und Film im Shakespeare-Unterricht. In: Schewe Manfred/Even Susanne: Scenario Jahrgang 2007. Ausgabe 01. ISSN $1649-8526$

Ministerium für Kultus, Jugend und Sport Baden-Württemberg (2004): Bildungsplan 2004 Realschule. In:

<http://www.bildung-staerktmenschen.de/service/downloads/ Bildungsplaene/Realschule/Realschule_Bildungsplan_Gesamt.pdf>, 31.05.2007

Ministerium für Kultus, Jugend und Sport Baden-Württemberg (2006): Bilingualer Unterricht. Lernen für Europa. Braunschweig: Westermann

Müller-Hartmann, Andreas/Grau, Maike (2004): Nur Tourist sein oder den Dialog wagen? Interkulturelles Lernen in der Begegnung. In: Der fremdsprachliche Unterricht 70, 2-11

Petersohn, Roland/Volkmann, Laurenz (Hrsg.) (2006a): Einleitung: Neue Perspektiven für den Shakespeare-Unterricht. In dies.: Shakespeare didaktisch I. Neue Perspektiven für den Unterricht. Tübingen, Stauffenburg Verlag, 9-20

Petersohn, Roland/Volkmann, Laurenz (Hrsg.) (2006b): Vorwort. In dies.: Shakespeare didaktisch I. Neue Perspektiven für den Unterricht. Tübingen, Stauffenburg Verlag, 7-8

Shakespeare, William, edited by T.J.B. Spencer (1996): Romeo and Juliet. London: Penguin Books, 49-168

Taylor, Michael (1996): Introduction. In: Shakespeare, William, edited by T.J.B. Spencer: Romeo and Juliet. London: Penguin Books, 7-44 


\section{Anhang}

Evaluation

\begin{tabular}{|llll|}
\hline 1. Das Ergebnis des & ETC-Projekts hat meine Erwartungen: \\
\hline O & 0 & $O$ & 0 \\
\hline Übertroffen & voll erfüllt & kaum erfüllt & nicht erfüllt \\
\hline 2. In folgenden Bereichen hat mir das & ETC-Projekt etwas gebracht: \\
\hline O & $O$ & $O$ & $O$ \\
\hline Theater & Englisch & Selbstbewusstsein & Organisation \\
\hline
\end{tabular}

\section{Diese Theatertechniken haben mir bei der Erarbeitung meiner Rolle ge-} holfen:

\begin{tabular}{|c|c|c|c|}
\hline O & 0 & O & 0 \\
\hline body language & voice & blocking /position & facial expression \\
\hline \multicolumn{4}{|c|}{ 4. Im Vergleich zum Englischunterricht habe ich ... Englisch gesprochen. } \\
\hline 0 & 0 & 0 & 0 \\
\hline sehr viel & viel & genauso viel & weniger \\
\hline \multicolumn{4}{|c|}{ 5. Insgesamt habe ich im Bereich „,Theater“... gelernt. } \\
\hline 0 & 0 & 0 & O \\
\hline sehr viel & viel & wenig & sehr wenig \\
\hline \multicolumn{4}{|c|}{ 6. Insgesamt habe ich im Bereich „,Englisch sprechen“... gelernt. } \\
\hline 0 & 0 & 0 & 0 \\
\hline sehr viel & viel & wenig & sehr wenig \\
\hline \multicolumn{4}{|c|}{ 7. Ich habe den Inhalt und Figurenkonstellation von R\&J ... verstanden. } \\
\hline 0 & 0 & 0 & O \\
\hline komplett & zum 7 & kaum & gar nicht \\
\hline
\end{tabular}

8. Im Vergleich zum Anfang konnte ich folgenden Gruppenprozess erkennen.

\begin{tabular}{|c|c|c|c|}
\hline 0 & 0 & 0 & 0 \\
\hline sehr hohe & hohe & kaum & keine \\
\hline Gruppendynamik & Gruppendynamik & Gruppendynamik & Gruppendynamik \\
\hline \multicolumn{4}{|c|}{ 9. Der Zeit-/Arbeitsaufwand für ETC hat sich gelohnt. } \\
\hline 0 & 0 & 0 & 0 \\
\hline ja, sehr & ja & nicht so sehr & gar nicht \\
\hline
\end{tabular}

10. Die Einteilung in Manager für die Organisation der Aufführung fand ich

\begin{tabular}{llll}
\hline sehr sinnvoll & 0 & 0 & 0 \\
\hline & sinnvoll & nicht zu sinnvoll & gar nicht sinnvoll
\end{tabular}

11. Die Einteilung der Stundenabläufe in Warm-up, Theatereinheit (body language, scene work etc.), Cool- down hat mir... gefallen.

\begin{tabular}{llll}
0 & 0 & 0 & 0 \\
\hline sehr gut & gut & nicht so sehr & gar nicht \\
\hline 12 & Das wollte
\end{tabular}

12. Das wollte ich noch sagen bzw. Verbesserungsvorschläge:

Auswertung der Evaluation (Schaubilder und freie Antworten)

12. Das wollte ich noch sagen:

- Es war ein sehr gelungenes Projekt und es hat mir sehr viel Spaß gemacht. 
Abbildung 7: Schaubild 1

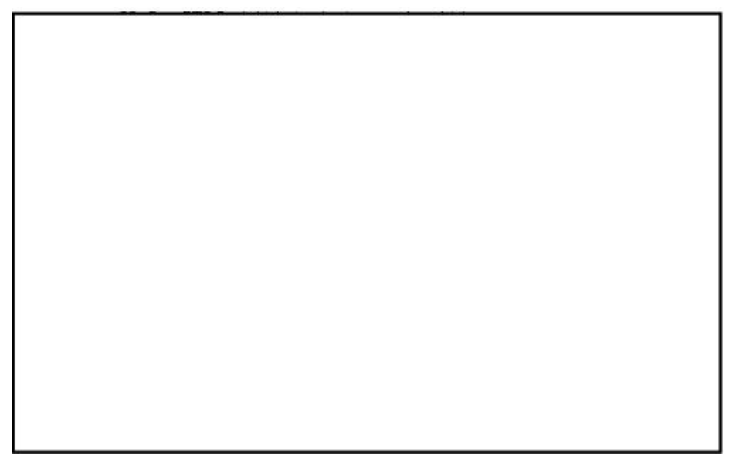

Abbildung 8: Schaubild 2

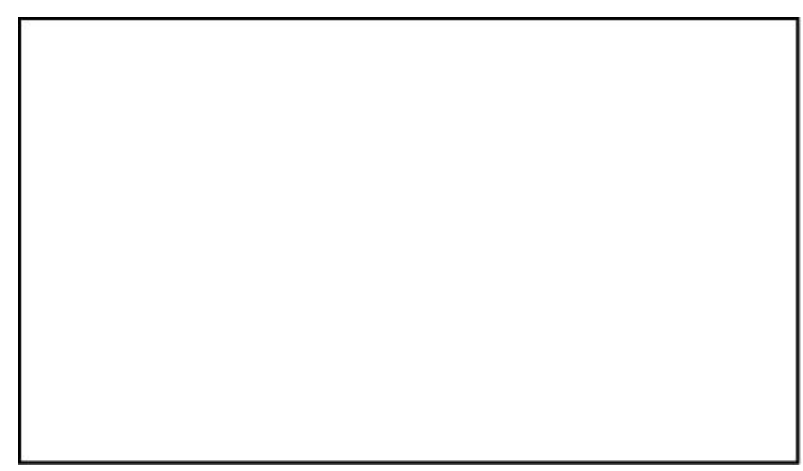

Abbildung 9: Schaubild 3

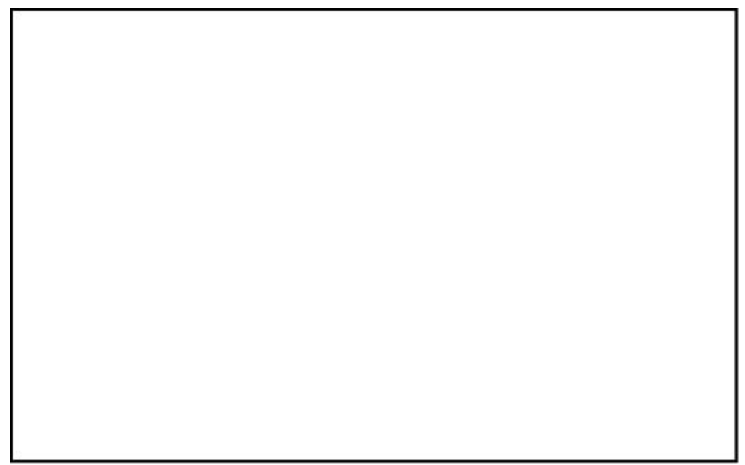

Abbildung 10: Schaubild 4 
Abbildung 11: Schaubild 5

Abbildung 12: Schaubild 6

Abbildung 13: Schaubild 7

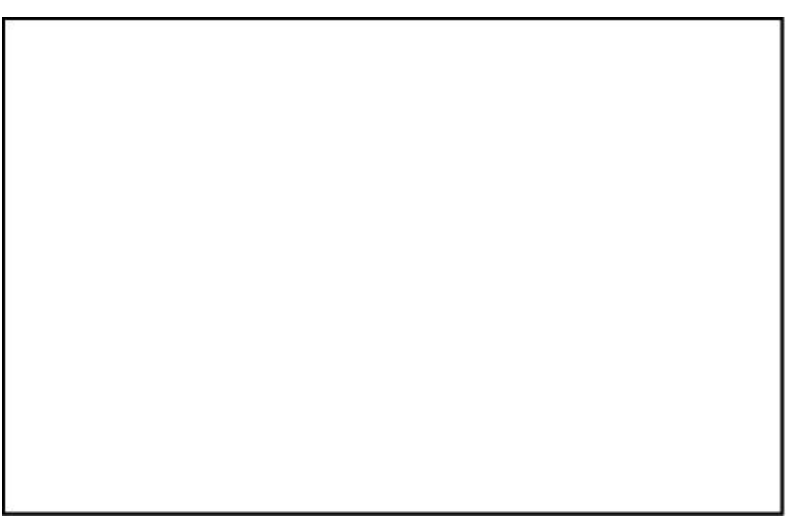

Abbildung 14: Schaubild 8 
Abbildung 15: Schaubild 9

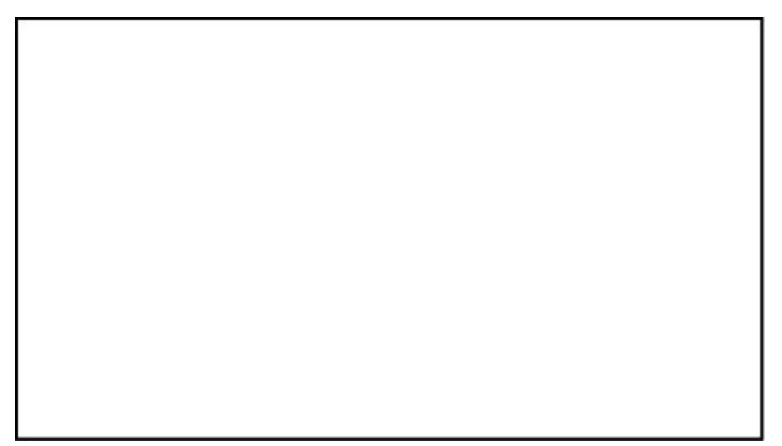

Abbildung 16: Schaubild 10

Abbildung 17: Schaubild 11

- Die Mitglieder sollten sich mehr Zeit nehmen bzw. bestimmte.

- In wenigen Momenten ein bisschen bessere Absprachen der actors.

- Mir hat es sehr gut gefallen.

- Die Show ist so gut gelungen wie ich es mir nie hätte vorstellen können!!!

- Aufführung war zu früh: erst später aufführen.

- Man sollte dafür sorgen, dass IMMER alle da sind (wenn möglich).

- Die Arbeit mit euch allen hat mir Spaß gemacht, und es war toll „auf der Bühne zu stehen“. 
- Es sollten die ETC-Members vielleicht noch mehr zuverlässig werden was Termine angeht.

- Ich würde auf alle Fälle wieder mitmachen wollen.

- Es hat super viel Spaß gemacht.

- Danke, Frau Passon. You did a great job.

- Es war eine tolle Zeit und es hat sich wirklich gelohnt auch am Wochenende zu kommen.

- War super so!

- Ich bin froh mitgemacht zu haben und würde es auch wieder tun, wenn wir nicht bald Prüfungen hätten.

- Es war einfach nur geil! 